\title{
Analytical and numerical study of Gauss-Bonnet holographic superconductors with Power-Maxwell field
}

\author{
Ahmad Sheykhi, ${ }^{a, b}$ Hamid Reza Salahi ${ }^{a}$ and Afshin Montakhab ${ }^{a}$ \\ ${ }^{a}$ Physics Department and Biruni Observatory, College of Sciences, Shiraz University, \\ Eram Square, P.O. Box 71454, Shiraz, Iran \\ ${ }^{b}$ Research Institute for Astronomy and Astrophysics of Maragha (RIAAM), \\ P.O. Box 55134-441, Maragha, Iran \\ E-mail: asheykhi@shirazu.ac.ir, salahi92.h@gmail.com, \\ montakhab@shirazu.ac.ir
}

ABSTRACT: We provide an analytical as well as a numerical study of the holographic $s$-wave superconductors in Gauss-Bonnet gravity with Power-Maxwell electrodynamics. We limit our study to the case where scalar and gauge fields do not have an effect on the background metric. We use a variational method, based on Sturm-Liouville eigenvalue problem for our analytical study, as well as a numerical shooting method in order to compare with our analytical results. Interestingly enough, we observe that unlike Born-Infeld-like nonlinear electrodynamics which decrease the critical temperature compared to the linear Maxwell field, the Power-Maxwell electrodynamics is able to increase the critical temperature of the holographic superconductors in the sublinear regime. We find that requiring the finite value for the gauge field on the asymptotic boundary $r \rightarrow \infty$, restricts the power parameter, $q$, of the Power-Maxwell field to be in the range $1 / 2<q<(d-1) / 2$. Our study indicates that it is quite possible to make condensation easier as $q$ decreases in its allowed range. We also find that for all values of $q$, the condensation can be affected by the Gauss-Bonnet coefficient $\alpha$. However, the presence of the Gauss-Bonnet term makes the transition slightly harder. Finally, we obtain an analytic expression for the order parameter and thus obtain the associated critical exponent near the phase transition. We find that the critical exponent has its universal value of $\beta=1 / 2$ regardless of the parameters $q, \alpha$ as well as dimension $d$, consistent with mean-field values obtained in previous studies.

KeYwords: AdS-CFT Correspondence, Black Holes

ARXIV EPRINT: 1603.00075 


\section{Contents}

1 Introduction 1

2 Gauss-Bonnet holographic superconductors with Power-Maxwell field 3

3 Critical temperature in terms of charge density 5

4 Critical temperature from numerical method $\quad 8$

$\begin{array}{llr}5 & \text { Critical exponent } & 10\end{array}$

6 Concluding remarks $\quad 13$

\section{Introduction}

It has been well established that the correspondence between gravity in a $d$-dimensional anti-de Sitter (AdS) spacetime and conformal field theory (CFT) living on the $(d-1)$ dimensional boundary of the corresponding spacetime, provides a powerful tool to study strongly coupled systems [1-3]. One of the intriguing applications of gauge/gravity duality is to shed light on the open problems in condensed-matter physics. Understanding the mechanism governing the high-temperature superconductor systems has been one of the most important challenges in condensed-matter physics. The AdS/CFT correspondence provides a new approach for calculating the properties of the superconductors using a dual classical gravity description and may shed light on the pairing mechanism in high temperature superconductors. Recently, it has been shown that some properties of strongly coupled superconductors can be potentially described by classical general relativity living in one higher dimension, which is known as holographic superconductors [4]. It was suggested that the instability of the bulk black hole corresponds to a second order phase transition from normal state to superconducting state which brings the spontaneous U(1) symmetry breaking [5]. The first holographic s-wave superconductor model known as Abelian-Higgs model was proposed by Hartnoll et al., [4, 6]. This basically consists of a black hole and a complex scalar field minimally coupled to an Abelian gauge field. It was observed that a scalar hair is formed below a certain critical temperature, $T_{c}$, due to the breaking of a local U(1) gauge symmetry near the black hole event horizon.

Following this novel idea by Hartnoll et al., [4], studies of holographic superconductors have attracted considerable attention and become an active field of research. Consequently, various aspects of the holographic superconductors, in the context of Einstein gravity have been explored in the literature [7-14]. Generalization to other gravity theories such as Gauss-Bonnet gravity have also been considered. For example, holographic superconductors with various condensates in Einstein-Gauss-Bonnet gravity were explored in [15]. A 
general class of holographic superconducting models with Gauss-Bonnet corrections terms was studied in [16]. It was shown that different values of Gauss-Bonnet correction term and model parameters can determine the order of phase transitions and critical exponents of second-order phase transition [16]. Also, using the variational method for the Sturm-Liouville eigenvalue problem, some properties of holographic superconductors with Gauss-Bonnet gravity in probe limit were analytically calculated [17]. It was found that the critical exponent of the condensation in Gauss-Bonnet gravity is $\beta=1 / 2$ which is consistent with mean-field theory and previous studies [17]. Other studies on the holographic superconductors in the framework of Gauss-Bonnet-AdS black holes were carried out in $[18-24]$.

Most of the works mentioned above have been done with the gauge field as a linear Maxwell field. However, it is interesting to investigate the effects of nonlinear electrodynamics on the properties of the holographic superconductor both in Einstein as well as Gauss-Bonnet gravities. Considering three types of nonlinear electrodynamics, namely, Born-Infeld, Logarithmic and Exponential nonlinear electrodynamics, and using numerical methods, it has been observed that, in the Schwarzschild AdS black hole background, the higher nonlinear electrodynamics corrections make the condensation harder [25, 26]. The effects of an external magnetic field on the holographic superconductors in the presence of nonlinear corrections to the usual Maxwell action were examined in [27, 28]. Analytical study of holographic superconductors in Born-Infeld electrodynamics have been carried out in [29-31]. When the background metric is the Gauss-Bonnet AdS black holes, several properties of holographic $s$-wave superconductors with Born-Infeld electrodynamics were explored by employing the Sturm-Liouville eigenvalue problem [32-34]. Performing explicit analytic computations, Meissner-like effect in the $(4+1)$-dimensional planar Gauss-BonnetAdS black hole background with several nonlinear corrections to the gauge field have also been studied [35, 36].

Applying the Sturm-Liouville analytical and numerical methods in the background of $d$-dimensional Schwarzschild AdS black hole, the behavior of the holographic superconductors have been explored by introducing a complex charged scalar field coupled with a Power-Maxwell field, both in the probe limit [37] and in the presence of backreaction [38]. However, the properties of the Gauss-Bonnet holographic superconductor from the PowerMaxwell field have not been explored yet. In this paper, we intend to extend the analytical study of holographic superconductor, by taking into account both the higher order GaussBonnet curvature correction terms, as well as the nonlinear Power-Maxwell electrodynamics. In particular, we shall disclose the effects of these correction terms on the critical temperature of the superconductor and its condensation. Interestingly, we find that the effect of sublinear Power-Maxwell field can lead to the easing of condensation formation and consequently a higher $T_{c}$.

This paper is outlined as follows: in the next section, we introduce the basic field equations of holographic superconductors in the background of Gauss-Bonnet AdS black holes when the gauge field is in the form of Power-Maxwell field. In section 3, we use the Sturm-Liouville method and obtain a relation between the critical temperature and charge density, and show that $T_{c}$ increases for some values of Power-Maxwell parameter. 
In section 4, we perform the numerical study and calculate the critical temperature by applying the shooting method. We also compare our analytical and numerical results in this section. In section 5 , we calculate the critical exponent and the condensation values of the Power-Maxwell holographic superconductor and show that it indeed has the universal value of $\beta=1 / 2$. The last section is devoted to concluding remarks.

\section{Gauss-Bonnet holographic superconductors with Power-Maxwell field}

We consider the action of Einstein-Gauss-Bonnet-AdS gravity in $d$-dimensions,

$$
S_{G}=\int d^{d} x \sqrt{-g}\left[(R-2 \Lambda)+\frac{\alpha}{2}\left(R^{2}-4 R^{\mu \nu} R_{\mu \nu}+R^{\mu \nu \rho \sigma} R_{\mu \nu \rho \sigma}\right],\right.
$$

where $\alpha$ is the Gauss-Bonnet coefficient, $\Lambda=-(d-1)(d-2) /\left(2 l^{2}\right)$ is the cosmological constant of $d$-dimensional AdS spacetime with radius $l$. For simplicity, hereafter we set $l=1$. Since we consider the probe limit, the gauge and the scalar fields do not back react on the background metric. The line element of the $d$-dimensional spacetime with flat horizon is written as [39]

$$
d s^{2}=-f(r) d t^{2}+\frac{d r^{2}}{f(r)}+r^{2} d x_{i} d x^{i}
$$

where

$$
f(r)=\frac{r^{2}}{2 \alpha}\left[1-\sqrt{1-4 \alpha\left(1-\frac{r_{+}^{d-1}}{r^{d-1}}\right)}\right],
$$

where $r_{+}$is the positive real root, $f\left(r_{+}\right)=0$. In the asymptotic region where $r \rightarrow \infty$, we have

$$
f(r) \approx \frac{r^{2}}{2 \alpha}(1-\sqrt{1-4 \alpha})
$$

Here, the positivity of $f(r)$ implies $0 \leq \alpha \leq 1 / 4$ and real. The Hawking temperature of black hole on the horizon $r_{+}$, which will be interpreted as the temperature of the CFT, may be obtained as [15]

$$
T=\frac{f^{\prime}\left(r_{+}\right)}{4 \pi}=\frac{(d-1) r_{+}}{4 \pi}
$$

where prime denotes derivative with respect to $r$. If we define the effective AdS radius as

$$
L_{\text {eff }}^{2}=\frac{2 \alpha}{1-\sqrt{1-4 \alpha}}
$$

then, in the asymptotic limit where $r \rightarrow \infty, f(r)$ can be written as

$$
f(r)=\frac{r^{2}}{L_{\text {eff }}^{2}}
$$

which has the form of Schwarzschild AdS black hole for $r \rightarrow \infty$. Now, consider the action of matter which is made of complex charged scalar field in bulk, that minimally couples to the Power-Maxwell field:

$$
S_{m}=\int d^{d} x \sqrt{-g}\left[-b\left(F_{\mu \nu} F^{\mu \nu}\right)^{q}-|\nabla \psi-i A \psi|^{2}-m^{2}|\psi|^{2}\right],
$$


where $F^{\mu \nu}$ is the electromagnetic field tensor, $A$ and $\psi$ are respectively, the gauge and complex charged scalar field. Here, $b$ is a constant and $q$ is the power parameter of the Power-Maxwell field. In the case where $b \rightarrow 1 / 4$ and $q \rightarrow 1$, the Power-Maxwell Lagrangian will reduce to the Maxwell case. Throughout this work, we set the charge of the scalar field $\psi$ equal to unity. Varying the matter action (2.8) with respect to the scalar and gauge fields yield the following field equations,

$$
\begin{aligned}
\left(\nabla_{\mu}-i A_{\mu}\right)\left(\nabla^{\mu}-i A^{\mu}\right) \psi-m^{2} \psi & =0, \\
4 b q \nabla_{\nu}\left[\left(F_{\sigma \delta} F^{\sigma \delta}\right)^{q-1} F^{\mu \nu}\right]-2 A^{\mu}|\psi|^{2}+i\left(\psi \nabla^{\mu} \psi^{*}-\psi^{*} \nabla^{\mu} \psi\right) & =0 .
\end{aligned}
$$

We shall assume $\psi$ and $\phi$ to be real and only function of $r$, i.e., $\psi=\psi(r)$ and $A=\phi(r) d t$. Thus, the equations of motion for the gauge and scalar fields can be written as,

$$
\begin{aligned}
\phi^{\prime \prime}+\left(\frac{d-2}{2 q-1}\right) \frac{\phi^{\prime}}{r}+\frac{\phi \psi^{2} \phi^{2-2 q}}{(-2)^{q} b q(2 q-1) f} & =0, \\
\psi^{\prime \prime}+\left(\frac{f^{\prime}}{f}+\frac{d-2}{r}\right) \psi^{\prime}+\left(\frac{\phi^{2}}{f^{2}}-\frac{m^{2}}{f}\right) \psi & =0 .
\end{aligned}
$$

Imposing the regularity conditions at the horizon $r_{+}$, yield

$$
\phi\left(r_{+}\right)=0, \quad f^{\prime}\left(r_{+}\right) \psi^{\prime}\left(r_{+}\right)=m^{2} \psi\left(r_{+}\right) .
$$

Now, we solve eqs. (2.11) and (2.12) in the asymptotic region $(r \rightarrow \infty)$. We find

$$
\begin{aligned}
& \phi \approx \mu-\frac{\rho^{\frac{1}{2 q-1}}}{r^{\frac{d-2}{2 q-1}-1}}, \\
& \psi \approx \frac{\psi^{-}}{r^{\lambda_{-}}}+\frac{\psi^{+}}{r^{\lambda_{+}}},
\end{aligned}
$$

where $\mu$ and $\rho$ are respectively interpreted as the chemical potential and charge density in the dual field theory, and

$$
\lambda_{ \pm}=\frac{1}{2}\left[(d-1) \pm \sqrt{(d-1)^{2}+4 m^{2} L_{\text {eff }}^{2}}\right] .
$$

According to AdS/CFT correspondence, $\psi_{+}$and $\psi_{-}$are normalizable modes of the scalar field $\psi$ which can be regarded as the source of the dual operator $\mathcal{O}, \psi_{-}=\left\langle\mathcal{O}_{-}\right\rangle$and $\psi_{+}=<\mathcal{O}_{+}>$, respectively. Following $[4,6]$, we can impose the boundary condition in which either $\psi_{+}$or $\psi_{-}$vanishes, so that the theory is stable in the asymptotic AdS region. It is worth noting that the gauge field $\phi$ depends on the power parameter $q$ of the Power-Maxwell field at the asymptotic AdS region $(r \rightarrow \infty)$. This is in contrast to the case of holographic superconductor with other nonlinear electrodynamics such as Born-Infeld [29-31] and Born-Infeld-like electrodynamics [25, 26].

At the boundary where $r \rightarrow \infty$, the gauge field should have a finite value. Thus eq. (2.14) implies that $\frac{d-2}{2 q-1}-1>0$, which restricts the values of $q$ to be $q<(d-1) / 2$. On the other hand since $\frac{d-2}{2 q-1}>1$, it must then be a positive real number, which considering that $d-2>0$, implies that $2 q-1>0$ or $q>1 / 2$. We have therefore simply extracted the meaningful range of the parameter $q$ to be $1 / 2<q<(d-1) / 2$. 


\section{Critical temperature in terms of charge density}

In this section, we obtain a relation between the critical temperature and charge density of the holographic superconductor in Gauss-Bonnet-AdS black holes in the presence of PowerMaxwell gauge field. We shall use the Sturm-Liouville eigenvalue problem and limit our study to the case where the scalar and gauge field do not affect on the background metric.

For this purpose, we first transform the coordinate $r$ to $z$, where $z=r_{+} / r$. In this new coordinate, the equations of motion (2.11) and (2.12) are rewritten as,

$$
\begin{aligned}
\phi^{\prime \prime}+\left(\frac{4 q-d}{2 q-1}\right) \frac{\phi^{\prime}}{z}+\frac{\phi \psi^{2} \phi^{2-2 q} r_{+}^{2 q}}{2^{q}(-1)^{3 q} b q(2 q-1) z^{4 q} f} & =0, \\
\psi^{\prime \prime}+\left(\frac{f^{\prime}}{f}-\frac{d-4}{z}\right) \psi^{\prime}+\frac{r_{+}^{2}}{z^{4}}\left(\frac{\phi^{2}}{f^{2}}-\frac{m^{2}}{f}\right) \psi & =0,
\end{aligned}
$$

where the prime now indicates the derivative with respect to the new coordinate $z$. Very close the critical temperature $T_{c}$, we have $\psi \rightarrow 0$, which implies that the condensation approaches zero. Therefore, eq. (3.1) reduces to

$$
\phi^{\prime \prime}+\left(\frac{4 q-d}{2 q-1}\right) \frac{\phi^{\prime}}{z}=0
$$

Using the boundary conditions (2.13) and (2.14), one can easily show that, near the critical temperature $\left(r_{+}=r_{+c}\right)$, eq. (3.3) has a solution of the form

$$
\phi=\zeta r_{+c}\left(1-z^{\frac{d-2}{2 q-1}-1}\right),
$$

where $\zeta=\left(\frac{\rho}{r_{+}^{d-2}}\right)^{\frac{1}{2 q-1}}$. Next, we introduce a trial function $F(z)$ as in [40] and rewrite the scalar field $\psi$, near the boundary,

$$
\left.\psi\right|_{z=0} \approx \frac{\psi_{i}}{r^{\lambda_{i}}}=<\mathcal{O}_{i}>\frac{z^{\lambda_{i}}}{r_{+}^{\lambda_{i}}} F(z)
$$

where $i=(+,-)$. The trial function near the boundary $z=0$ satisfies the boundary conditions $F(0)=1$ and $F^{\prime}(0)=0$ [40]. Substituting eqs. (2.3), (3.4) and (3.5), in eq. (3.2), one obtains

$$
\begin{aligned}
F^{\prime \prime}(z)+ & \left\{\frac{2 \lambda_{i}}{z}+\frac{-2(d-3) z^{d-1} \alpha+(d-2)\left(-1+4 \alpha+\sqrt{1+4\left(z^{d-1}-1\right) \alpha}\right)}{z \sqrt{1+4\left(z^{d-1}-1\right) \alpha}\left(-1+\sqrt{1+4\left(z^{d-1}-1\right) \alpha}\right)}\right\} F^{\prime}(z) \\
+ & \left\{\frac{4\left(-1+z^{\frac{d-2 q-1}{2 q-1}}\right) \alpha^{2} \zeta^{2}}{\left(-1+\sqrt{1+4\left(z^{4}-1\right) \alpha}\right)^{2}}+\frac{\lambda_{i}\left(\lambda_{i}-1\right)}{z^{2}}+\frac{2 m^{2} \alpha}{z^{2}\left(-1+\sqrt{1+4\left(z^{d-1}-1\right)}\right)}\right. \\
& \left.-\frac{\left(2(d-3) z^{d-1} \alpha+(d-2)\left(-1+\sqrt{1+4\left(z^{d-1}-1\right) \alpha}\right) \lambda_{i}\right.}{z^{2} \sqrt{1+4\left(z^{d-1}-1\right) \alpha}\left(-1+\sqrt{1+4\left(z^{d-1}-1\right) \alpha}\right)}\right\} F(z)=0 .
\end{aligned}
$$


The above equation is a second order ordinary differential equation in the form of

$$
F^{\prime \prime}(z)+p(z) F^{\prime}(z)+q(z) F(z)+\zeta^{2} w(z) F(z)=0,
$$

where

$$
\begin{aligned}
& p(z)=\frac{2 \lambda_{i}}{z}+\frac{-2(d-3) z^{d-1} \alpha+(d-2)\left(-1+4 \alpha+\sqrt{1+4\left(z^{d-1}-1\right) \alpha}\right)}{z \sqrt{1+4\left(z^{d-1}-1\right) \alpha}\left(-1+\sqrt{1+4\left(z^{d-1}-1\right) \alpha}\right)}, \\
& q(z)=\frac{\lambda_{i}\left(\lambda_{i}-1\right)}{z^{2}}-\frac{\left(2(d-3) z^{d-1} \alpha+(d-2)\left(-1+\sqrt{1+4\left(z^{d-1}-1\right) \alpha}\right) \lambda_{i}\right.}{z^{2} \sqrt{1+4\left(z^{d-1}-1\right) \alpha}\left(-1+\sqrt{1+4\left(z^{d-1}-1\right) \alpha}\right)} \\
& +\frac{2 m^{2} \alpha}{z^{2}\left[-1+\sqrt{1+4\left(z^{d-1}-1\right)}\right]} \\
& w(z)=\frac{4\left(-1+z^{\frac{d-2 q-1}{2 q-1}}\right) \alpha^{2} \zeta^{2}}{\left[-1+\sqrt{1+4\left(z^{4}-1\right) \alpha}\right]^{2}} .
\end{aligned}
$$

It is a matter of calculations to convert eq. (3.6) to the standard form of the Sturm-Liouville equation,

$$
\left[T(z) F^{\prime}(z)\right]^{\prime}-Q(z) F(z)+\zeta^{2} P(z) F(z)=0
$$

where

$$
\begin{aligned}
& Q(z)=-T(z) q(z), \quad P(z)=T(z) w(z) \\
& T(z)=z^{2 \lambda+2-d} \sqrt{1-z^{d-1}} \exp \left\{-\frac{z^{d-1} \tilde{F}_{1}\left(1, \frac{1}{2}, 1,2 ; \frac{4 z^{d-1} \alpha}{4 \alpha-1}, z^{d-1}\right)}{2 \sqrt{1-4 \alpha}}\right\} .
\end{aligned}
$$

Here $\tilde{F}_{1}$ is the Appell hypergeometric function [41] with two variables $\alpha$ and $z$. Expanding eq. (3.12) for small $\alpha$ and keeping terms up to $\mathcal{O}\left(\alpha^{2}\right)$, we get

$$
\begin{aligned}
& T(z) \approx z^{2 \lambda+2-d}\left(z^{d-1}-1\right)\left\{1-\alpha z^{d-1}+\alpha^{2}\left(2 z^{2 d-2}-3 z^{d-1}\right)+\mathcal{O}\left(\alpha^{3}\right)\right\}, \\
& Q(z) \approx-z^{2 \lambda-d}\left\{\lambda \left[(d-\lambda-1)+2 \alpha^{2}(2 d+\lambda-2) z^{3 d-3}+\left(3 \alpha^{2}+\alpha+1\right) \lambda z^{d-1}\right.\right. \\
& \left.\left.-\alpha(5 \alpha+1)(d+\lambda-1) z^{2 d-2}\right]+\tilde{m}^{2}\right\} \\
& P(z) \approx-\frac{z^{\frac{d(3-2 q)}{2 q-1}+2 \lambda}}{z^{d-1}-1}\left(z^{\frac{d}{1-2 q}}-z^{\frac{2 q+1}{1-2 q}}\right)^{2}\left\{\alpha^{2} z^{2 d}-\alpha(\alpha+1) z^{d+1}+[\alpha(\alpha+2)-1] z^{2}\right\} .
\end{aligned}
$$

We have also taken $\tilde{m}^{2}=m^{2} L_{\text {eff }}^{2}$ where $L_{\text {eff }}$ is given by eq. (2.6). According to the Sturm-Liouville eigenvalue problem, the eigenvalues of eq. (3.11) are obtained as

$$
\zeta^{2}=\frac{\int_{0}^{1}\left[T(z)\left[F^{\prime}(z)\right]^{2}+Q(z) F^{2}(z)\right] d z}{\int_{0}^{1} T(z) F^{2}(z) d z} .
$$

Our strategy is to obtain the minimum value of $\zeta^{2}$ by varying eq. (3.17). Let us choose the trial function as $F(z)=1-a z^{2}$ which satisfies the boundary conditions. In the remaining part of this section we shall concentrate on $\lambda_{+}$. 


\begin{tabular}{|c|c|c|c|c|c|c|c|c|}
\hline & \multicolumn{2}{|c|}{$\alpha=0.05$} & \multicolumn{2}{c|}{$\alpha=0.1$} & \multicolumn{2}{c|}{$\alpha=0.15$} & \multicolumn{2}{c|}{$\alpha=0.2$} \\
\hline$q$ & $a$ & $\zeta_{\min }^{2}$ & $a$ & $\zeta_{\min }^{2}$ & $a$ & $\zeta_{\min }^{2}$ & $a$ & $\zeta_{\min }^{2}$ \\
\hline $5 / 4$ & 0.7644 & 47.7162 & 0.7579 & 53.1425 & 0.7488 & 60.3734 & 0.7358 & 70.5134 \\
\hline 1 & 0.7146 & 19.9456 & 0.7050 & 22.1278 & 0.6917 & 25.0053 & 0.6730 & 28.9837 \\
\hline $3 / 4$ & 0.5878 & 8.6847 & 0.5693 & 9.5348 & 0.5442 & 10.6247 & 0.5092 & 12.0771 \\
\hline
\end{tabular}

Table 1. Analytical results for $\zeta_{\min }^{2}$ and $a$ with different values of the power parameter $q$ and Gauss-Bonnet parameter $\alpha$ for $\lambda_{+}$. Here we have taken $d=5$ and $\tilde{m}^{2}=-3$.

Calculating the integrals in eq. (3.17) for $d=5, q=1, \tilde{m}^{2}=-3$, and $\alpha=0.1$ we get

$$
\zeta^{2}=\frac{128.654 a-199.113}{a(a-3.4531)+3.6591}+85.0957
$$

which has a minimum $\zeta_{\min }^{2}=22.1278$ for $a=0.7050$. By choosing $d=5, q=3 / 4, \tilde{m}^{2}=-3$ and $\alpha=0.2$, for $\zeta^{2}$ we arrive at

$$
\zeta^{2}=\frac{28.096 a-33.1738}{a(1 . a-3.04098)+2.64731}+25.9692 .
$$

The minimum will now be $\zeta_{\min }^{2}=12.0771$ at $a=0.5092$. We summarize our results for $\zeta_{\min }^{2}$ in table 1.

Next, we calculate the critical temperature. At the critical point we have $r_{+}=r_{+c}$, eq. (2.5) leads to

$$
T_{c}=\frac{(d-1) r_{+c}}{4 \pi} .
$$

Using the fact that $\zeta_{\min }=\left(\frac{\rho}{r_{+c}^{d-2}}\right)^{\frac{1}{2 q-1}}$, we finally reach

$$
T_{c}=\gamma \rho^{\frac{1}{d-2}}, \quad \gamma=\frac{(d-1)}{4 \pi} \zeta_{\min }^{\frac{1-2 q}{d-2}} .
$$

We now provide a systematic presentation of the critical temperature as a function of various variables and parameters. The critical temperature can change continuously as a function of $q$ in the allowed range. Therefore, in figure 1 we provide such a variation for three different values of $\alpha$ and two different values of $\tilde{m}^{2}$ for $d=5$. Clearly, increasing $\alpha$ decreases the critical temperature. However, and more importantly, the critical temperature is a decreasing function of $q$, indicating the possibility of easing the superconducting phase transition with sublinear Power-Maxwell field. It is also interesting to note that the behavior of the critical temperature at both lower and upper bound of $q$. In the lower bound, where the maximum occurs, the results are independent of both $\alpha$ as well as $\tilde{m}^{2}$. This can clearly be seen from eq. (3.21) where $\gamma$ becomes only a function of $d$ in this limit. On the other hand, the superconducting phase transition vanishes as $q$ approaches its upper bound. This can also be seen from eq. (3.4) where the gauge field vanishes for $q \rightarrow(d-1) / 2$, where $\psi=0$ becomes the only solution. This is consistent with the understanding that the electrostatic repulsion must overcome the gravitational attraction for 


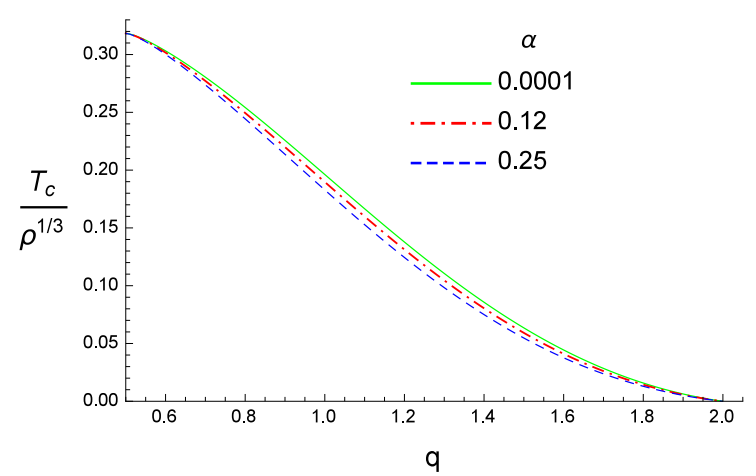

(a) $\tilde{m}^{2}=-3$

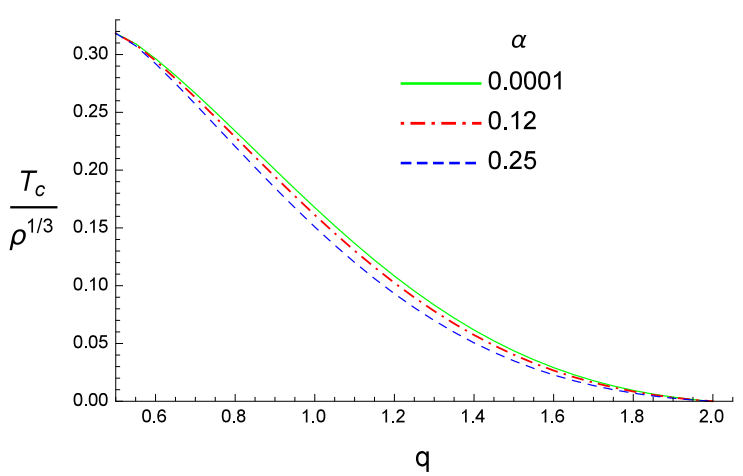

(b) $\tilde{m}^{2}=0$

Figure 1. Rescaled critical temperature $\left(T_{c} / \rho^{1 / 3}\right)$ as a function of $q$ for $d=5$.

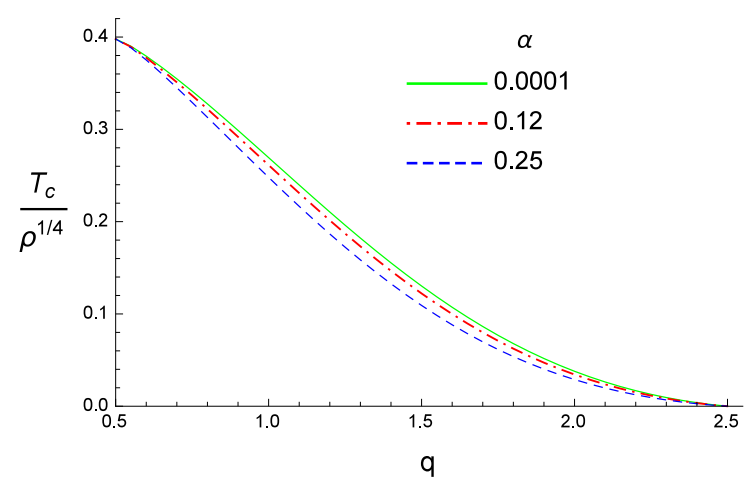

(a) $\tilde{m}^{2}=-4$

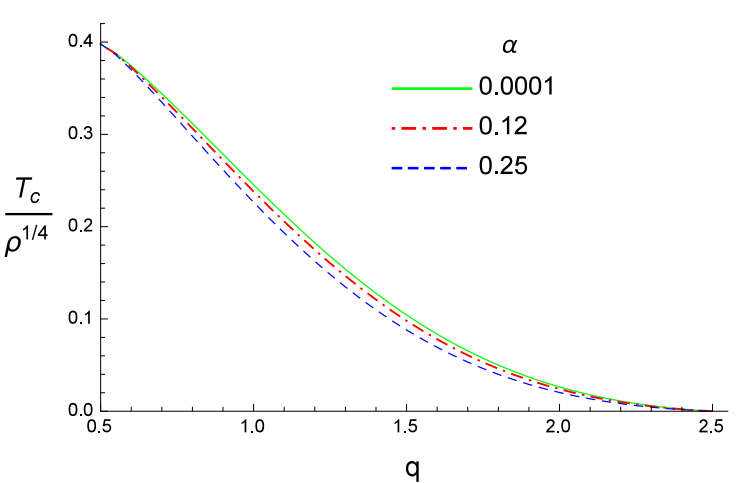

(b) $\tilde{m}^{2}=0$

Figure 2. Rescaled critical temperature $\left(T_{c} / \rho^{1 / 4}\right)$ as a function of $q$ for $d=6$.

the formation of the scalar field [42]. We also provide the same information in one higher dimensions, $d=6$, in figure 2 , where the critical temperature now scales with $\rho^{1 / 4}$. Here, the general trend discussed above is the same except that the range of possible $q$ values has increased and that the transition point at the lower bound $(q=1 / 2)$ increases with increasing $d$. This is also consistent with the general understanding that phase transitions are easier to achieve in higher dimensional systems.

In order to more explicitly show the effect of $\tilde{m}^{2}$, figure 3 provides such a plot for (a) $d=5$ and (b) $d=6$, where one can clearly see that decreasing $\tilde{m}^{2}$ increases the critical temperature for a given value of $q$. Furthermore, figure 4 shows the easing of superconducting phase transition in a higher dimensional system.

\section{Critical temperature from numerical method}

In this section, we propose to numerically study the critical behavior of the Power-Maxwell holographic superconductor in Gauss-Bonnet gravity. For this purpose, we adopt the shooting method [43]. Let us note that eqs. (2.11) and (2.12) remain unchanged under transformation

$$
r \rightarrow a r, \quad f \rightarrow a^{2} f, \quad \phi \rightarrow a \phi, \quad \psi \rightarrow \psi
$$




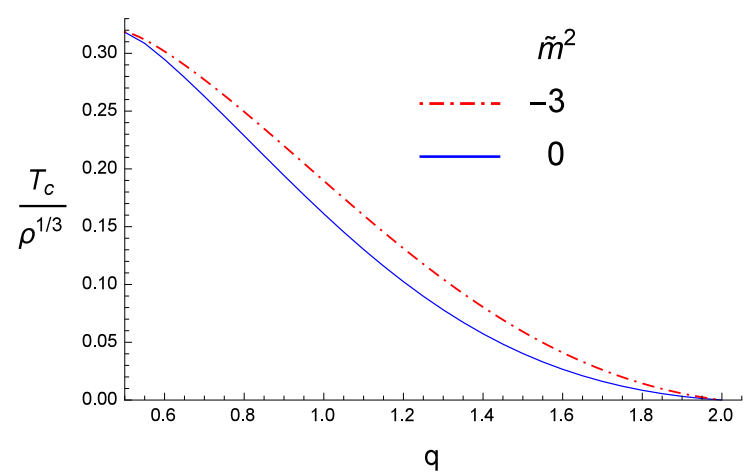

(a) $\mathrm{d}=5$

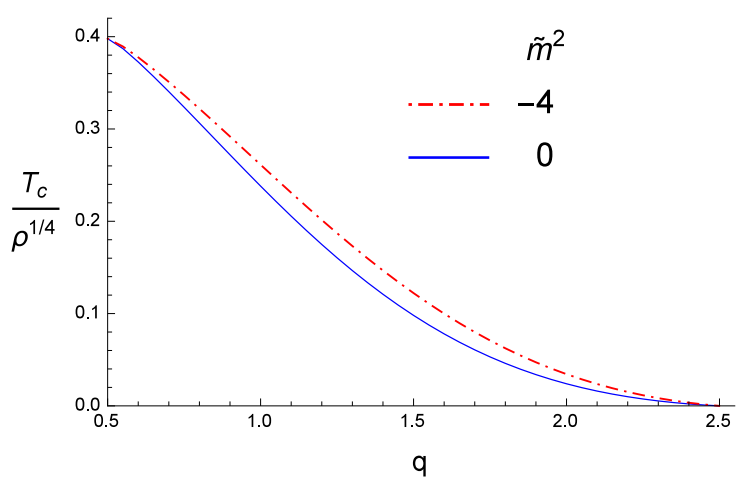

(b) $d=6$

Figure 3. Rescaled critical temperature $\left(T_{c} / \rho^{1 /(d-2)}\right)$ as a function of $q$ for a fixed value of $\alpha=0.12$.

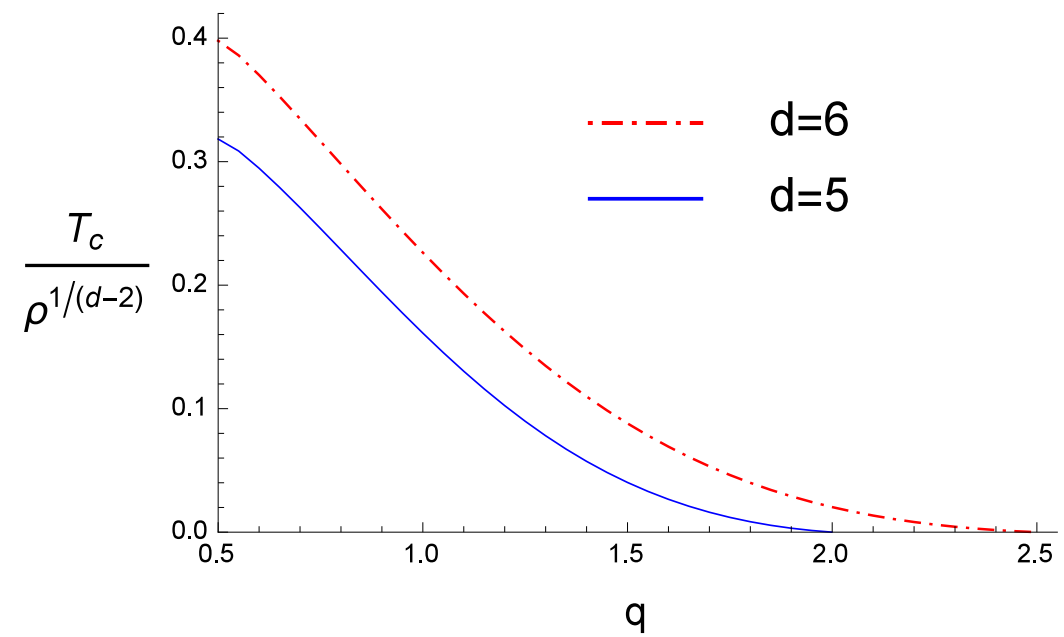

Figure 4. Rescaled critical temperature $\left(T_{c} / \rho^{1 /(d-2)}\right)$ as a function of $q$ for a fixed value of $\tilde{m}^{2}=0$ and $\alpha=0.12$.

Taking advantages of this scaling symmetry, we can set $r_{+}$as unity in our numerical calculations and we can build dimensionless quantities such as $T / \rho^{\frac{1}{d-2}}$.

Because we want to obtain critical temperature, we will take $\phi$ as (3.4). Thus the equation of motion for $\psi$ in the $z$ coordinate becomes

$$
\psi^{\prime \prime}+\left(\frac{f^{\prime}}{f}-\frac{d-4}{z}\right) \psi^{\prime}+\frac{1}{z^{4}}\left(\frac{\rho^{\frac{2}{2 q-1}}\left(1-z^{\frac{d-2}{2 q-1}-1}\right)^{2}}{f^{2}}-\frac{m^{2}}{f}\right) \psi=0 .
$$

The behavior of $\psi$ near the horizon boundary is

$$
\psi \approx \psi(1)+\psi^{\prime}(1)(1-z)+\frac{\psi^{\prime \prime}(1)}{2}(1-z)^{2}+\ldots,
$$

and near the infinite boundary is

$$
\psi \approx \psi^{-} z^{\lambda_{-}}+\psi^{+} z^{\lambda_{+}} .
$$




\begin{tabular}{|c|c|c|c|c|c|c|}
\hline & \multicolumn{2}{|c|}{$\alpha=0.05$} & \multicolumn{2}{c|}{$\alpha=0.1$} & \multicolumn{2}{c|}{$\alpha=0.15$} \\
\hline$q$ & Analytical & Numerical & Analytical & Numerical & Analytical & Numerical \\
\hline $5 / 4$ & $0.1211 \rho^{1 / 3}$ & $0.1248 \rho^{1 / 3}$ & $0.1179 \rho^{1 / 3}$ & $0.1212 \rho^{1 / 3}$ & $0.1142 \rho^{1 / 3}$ & $0.1169 \rho^{1 / 3}$ \\
\hline 1 & $0.1933 \rho^{1 / 3}$ & $0.1949 \rho^{1 / 3}$ & $0.1900 \rho^{1 / 3}$ & $0.1914 \rho^{1 / 3}$ & $0.1861 \rho^{1 / 3}$ & $0.1871 \rho^{1 / 3}$ \\
\hline $3 / 4$ & $0.2658 \rho^{1 / 3}$ & $0.2659 \rho^{1 / 3}$ & $0.2638 \rho^{1 / 3}$ & $0.2638 \rho^{1 / 3}$ & $0.2614 \rho^{1 / 3}$ & $0.2614 \rho^{1 / 3}$ \\
\hline
\end{tabular}

Table 2. Comparison of analytical and numerical values of critical temperature for $d=5$ and $\tilde{m}^{2}=-3$ for various values of $q$ and $\alpha$.

Using the equation of motion for $\psi$, we can find $\psi^{\prime}(1)$ and $\psi^{\prime \prime}(1)$ in the terms of $\psi(1)$ which is an arbitrary constant. Considering the fact that we are close to the critical point and $\psi$ is very small, we choose $\psi(1)=0.001$. According to the shooting method, for specific dimension $d$ and reduced scalar field mass $\tilde{m}^{2}$, we can perform numerical calculation near the horizon boundary with one shooting parameter $\rho$ to get proper solutions at the infinite boundary. For specific values of $\rho$, boundary condition $\psi_{-}=0$ (or $\psi_{+}=0$ ) is satisfied. In figure 5 , we plot $\psi(z)$ for three first critical charge densities for some values of $q, \tilde{m}^{2}$ and $\alpha$ in five dimensions. It is believed that the first condensation occurs for the lowest value of $\rho_{c}$ and condensations due to higher values of $\rho_{c}$ are not considered to be stable [42]. Having the lowest $\rho_{c}$ and using dimensionless quantity $T / \rho^{\frac{1}{d-2}}$, we easily obtain the critical temperature for different values of $q$ and $\alpha$. In table 2 we compare the values of the critical temperature from the numerical results based on shooting method with boundary condition $\psi_{-}=0$ for $\tilde{m}^{2}=-3$ with the analytical results obtained in the previous section. We see that our analytical results obtained by Sturm-Liouville method are in good agreement with the numerical results from shooting method.

\section{Critical exponent}

In this section, we propose to analytically calculate the critical exponent of the GaussBonnet holographic superconductor in the presence of the Power-Maxwell field. Near the critical temperature, $T \approx T_{c}$, the condensation value $\left\langle\mathcal{O}_{i}\right\rangle$ is very small. Inserting eq. (3.5) into eq. (3.1), we get

$$
\phi^{\prime \prime}+\left(\frac{4 q-d}{2 q-1}\right) \frac{1}{z} \phi^{\prime}+\frac{r_{+}^{2 q-2 \lambda_{i}-2} z^{2 \lambda_{i}-4 q} F^{2} \phi^{2-2 q} \phi<\mathcal{O}_{i}>^{2}}{(-1)^{3 q} 2^{q} b q(2 q-1) g(z)}=0
$$

where $g(z)=\frac{1-\sqrt{1-4 \alpha\left(1-z^{d-1}\right)}}{2 \alpha z^{2}}$. One can easily see that for $\left\langle\mathcal{O}_{i}\right\rangle=0$, eq. (5.1) has a solution of the form (3.4). Because $\left\langle\mathcal{O}_{i}\right\rangle$ is very small, we assume that solution of eq. (5.1) has the form

$$
\phi(z)=A T_{c}\left(1-z^{\frac{d-2}{2 q-1}-1}\right)-\left(A T_{c}\right)^{p}\left[\frac{r_{+}^{2 q-2 \lambda_{i}-2}<\mathcal{O}_{i}>^{2}}{(-1)^{3 q} 2^{q} b q(2 q-1)}\right] \chi(z)
$$




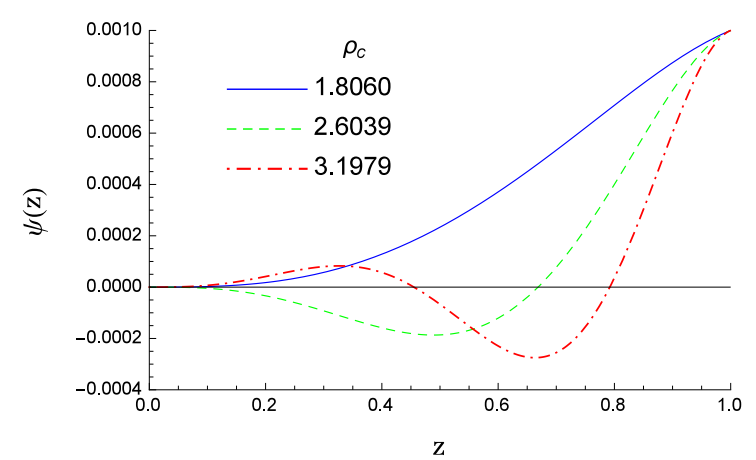

(a) $q=3 / 4, \alpha=0.05, \tilde{m}^{2}=-3$

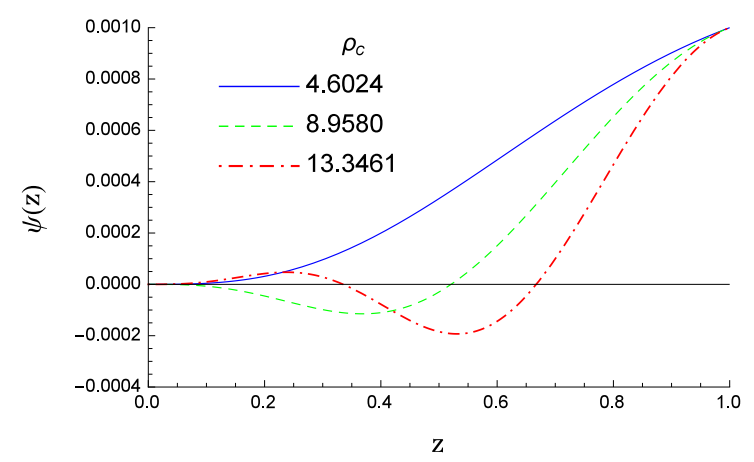

(c) $q=1, \alpha=0.1, \tilde{m}^{2}=-3$

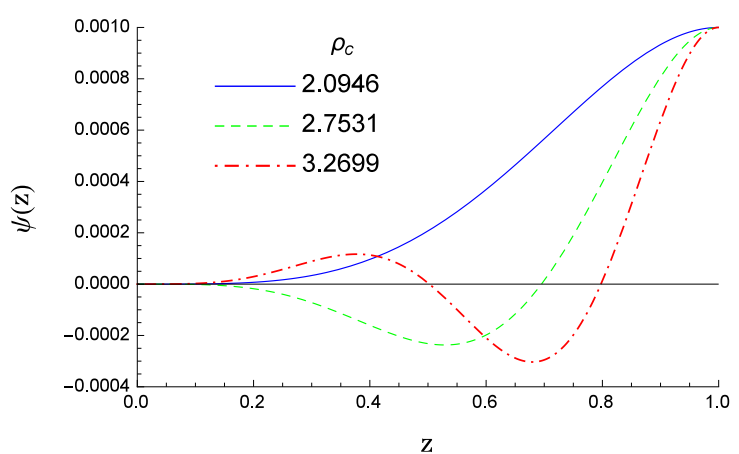

(b) $q=3 / 4, \alpha=0.15, \tilde{m}^{2}=0$

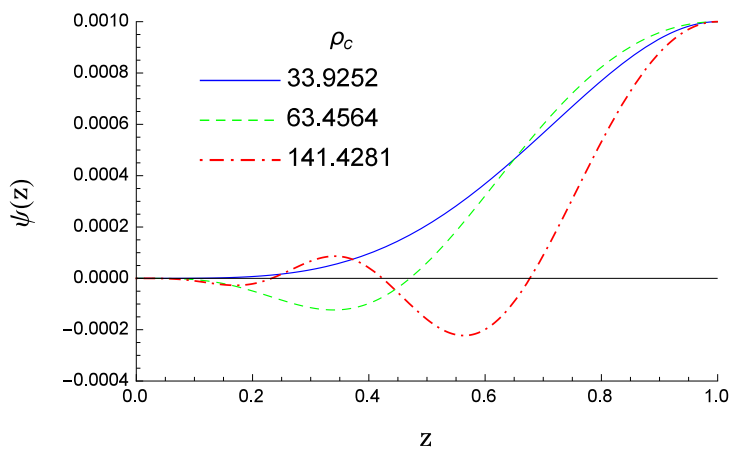

(d) $q=5 / 4, \alpha=0.05, \tilde{m}^{2}=0$

Figure 5. $\psi(z)$ for some values of $q, \alpha$ and $\tilde{m}^{2}$ in five dimensions with boundary condition $\psi^{-}=0$ for three lowest values of critical charge density. In each of these graphs, blue line shows the lowest-lying $\rho_{c}$.

where $A=\frac{4 \pi}{d-1} \zeta_{\min }$. Substituting eq. (5.2) into eq. (5.1) and keeping terms up to the linear order in $\alpha$, we get

$$
\chi^{\prime \prime}+\left(\frac{4 q-d}{2 q-1}\right) \frac{\chi^{\prime}}{z}-\frac{\left(1-\frac{d-2}{2 q-1}\right)^{2-2 q} z^{\eta}\left(1-z^{\frac{d-2}{2 q-1}-1}\right) F(z)^{2}}{\left(z^{4}-1\right)\left[\left(z^{4}-1\right) \alpha-1\right]}=0,
$$

where

$$
\eta=2 \lambda-4 q+\left(\frac{d-2}{2 q-1}-2\right)(2-2 q)+2
$$

and we have set $p=3-2 q$. Note that this choice for $p$ allows us to obtain an equation for $\chi$ independent of $T_{c}$ and $A$. Near the boundary, the solution of $\phi$ given in eq. (2.14), after using $r=r_{+} / z$, can be written as

$$
\phi(z)=\mu-\frac{\rho^{\frac{1}{2 q-1}}}{r_{+}^{\frac{d-2}{2 q-1}-1}} z^{\frac{d-2}{2 q-1}-1} .
$$

On the boundary $(z=0)$, eq. (5.5) implies that $\phi(z=0)=\mu$. According to [38], we have

$$
\mu=\frac{\rho^{\frac{1}{2 q-1}}}{r_{+}^{\frac{d-2}{2 q-1}-1}} .
$$


Combining eqs. (2.5), (3.21), (5.5) and (5.6), we can find

$$
\phi(z=0)=A \frac{T_{c}^{\frac{d-2}{2 q-1}}}{T^{\frac{d-2}{2 q-1}-1}} .
$$

We note that eq. (5.2) is valid for all $z$ including $z=0$. Therefore by equating eqs. (5.7) and (5.2) at the boundary $(z=0)$ to find

$$
A \frac{T_{c}^{\frac{d-2}{2 q-1}}}{T^{\frac{d-2}{2 q-1}-1}}=A T_{c}-\left(A T_{c}\right)^{3-2 q}\left[\frac{r_{+}^{2 q-2 \lambda_{i}-2}<\mathcal{O}_{i}>^{2}}{(-1)^{3 q} 2^{q} b q(2 q-1)}\right] \chi(0) .
$$

First of all, we note that the solutions of eq. (5.3) for $\chi(z)$ depend on the parameters $d, q$ and $\lambda_{i}$ but are independent of $r_{+}, T_{c}$ and $T$. Eq. (5.3) may not have an analytical solution for all values of parameters $q, d$ and $\lambda_{i}$ but we only need $\left.\chi\right|_{z \rightarrow 0}$ which can be found numerically using the boundary conditions $\chi(1)=\chi^{\prime}(1)=0$ [45]. Thus, $\chi(z=0)$ is just a constant which does not include $r_{+}, T_{c}$ or $T$. Solving eq. (5.8) for $\left\langle\mathcal{O}_{i}\right\rangle$, after using the fact that $\chi(0)$ is a constant, one finds

$$
A T_{c}\left[\left(\frac{T_{c}}{T}\right)^{\frac{d-2}{2 q-1}-1}-1\right]=s T_{c}^{3-2 q} T^{2 q-2 \lambda_{i}-2}<\mathcal{O}_{i}>^{2},
$$

where

$$
s=\frac{A^{3-2 q} \chi(0)}{(-1)^{3 q-1} 2^{q} b q(2 q-1)}\left(\frac{4 \pi}{d-1}\right)^{2 q-2 \lambda-2},
$$

is a constant independent of $r_{+}$and $T_{c}$. Eq. (5.9) can be rearranged as

$$
A\left[\left(\frac{T_{c}}{T}\right)^{\frac{d-2}{2 q-1}-1}-1\right]=s\left(\frac{T_{c}}{T}\right)^{2 \lambda_{i}-2 q-2} \frac{<\mathcal{O}_{i}>^{2}}{T_{c}^{2 \lambda_{i}}} .
$$

Finally, the explicit expression for $\left\langle\mathcal{O}_{i}\right\rangle$ is obtained as

$$
<\mathcal{O}_{i}>=\tilde{A} T_{c}^{\lambda_{i}}\left(\frac{T}{T_{c}}\right)^{\lambda_{i}-q-1} \sqrt{\left(\frac{T_{c}}{T}\right)^{\frac{d-2}{2 q-1}-1}\left[1-\left(\frac{T}{T_{c}}\right)^{\frac{d-2}{2 q-1}-1}\right]},
$$

where $\tilde{A}=A / s$. Near the critical temperature $T \approx T_{c}$, we can write $\frac{T}{T_{c}} \approx \frac{T_{c}}{T} \approx 1$, and then $\left\langle\mathcal{O}_{i}\right\rangle$ reduces to

$$
<\mathcal{O}_{i}>\approx \tilde{A} T_{c}^{\lambda_{i}} \sqrt{1-\left(\frac{T}{T_{c}}\right)^{\frac{d-2}{2 q-1}-1}} .
$$

Using the fact that near the critical temperature, $t=1-T / T_{c}$ is very small, we can rewrite the above as

$$
<\mathcal{O}_{i}>\approx \tilde{A} T_{c}^{\lambda_{i}} \sqrt{1-\left[1-\left(\frac{d-2}{2 q-1}-1\right) t\right]} \approx \tilde{A} T_{c}^{\lambda_{i}} \sqrt{\left(\frac{d-2}{2 q-1}-1\right) t}
$$


It is worth noting that the result (5.14) holds for both the scalar operators $\left\langle\mathcal{O}_{+}\right\rangle$and $<\mathcal{O}_{-}>$in the allowed ranges of the Power-Maxwell parameter $1 / 2<q<(d-1) / 2$ and in arbitrary dimensions. It is obvious from (5.14) that the critical exponent of system is equal to $\beta=1 / 2$ which is consistent with the mean-field value. This implies that neither the higher curvature Gauss-Bonnet correction term nor the Power-Maxwell nonlinear electrodynamics affect the critical exponent of the holographic superconductors. This is not surprising as the critical exponent is a universal feature of such theories. However, the nonuniversal proportionality constant $\left(\tilde{A} T_{c}^{\lambda_{i}} \sqrt{\left(\frac{d-2}{2 q-1}-1\right)}\right)$ depends on $d$ as well as $q$. We note that the behavior of this proportionally constant is somewhat suspect at $q=1 / 2$. This is not an allowed value, but we can get close to it arbitrarily. The more exact formula for order parameter, eq. (5.12), also display some limitation for $q \rightarrow 1 / 2$ and/or $T \rightarrow 0$ limit.

\section{Concluding remarks}

Based on the Sturm-Liouville eigenvalue problem, we analytically investigated the holographic $s$-wave superconductors with Power-Maxwell electrodynamics in the background of Gauss-Bonnet AdS spacetime. Our main results are analytic expressions for the critical temperature and order parameter (which gives the critical exponent) associated with the superconducting phase transition. We provided a systematic illustration of the behavior of the critical temperature as various parameters change. We also carried out a numerical study of the critical temperature by using the shooting method. We confirmed that the analytical results obtained based on the Sturm-Liouville method are in good agreement with the numerical results. The physically relevant range of Power-Maxwell parameter $1 / 2<q<(d-1) / 2$ is in focus here. Most importantly, and in contrast to various other studies, we find that the presence of nonlinear electrodynamics can lead to the easing of the transition thus leading to higher critical temperatures for sublinear Power-Maxwell parameter $q$. On the other hand, the effect of Gauss-Bonnet coefficient $\alpha$ always lowers the critical temperature. Also, transition is helped in higher dimensions. We find that the critical exponent associated with the order parameter is not effected by the main parameter under consideration here, i.e, $q, \alpha$ and $d$.

Finally, we would like to mention that in this paper, we only considered the probe limit, where the scalar and gauge fields do not back react on the metric background. It would be interesting if one could consider the Gauss-Bonnet holographic superconductor from PowerMaxwell field away from the probe limit and take the backreaction into account. One may also consider the case in which the Gauss-Bonnet coupling $\alpha$ is negative [46]. These issues are currently under investigation and the results will be reported subsequently.

\section{Acknowledgments}

We thank Shiraz University Research Council. The work of A.S has been supported financially by Research Institute for Astronomy and Astrophysics of Maragha (RIAAM), Iran. 
Open Access. This article is distributed under the terms of the Creative Commons Attribution License (CC-BY 4.0), which permits any use, distribution and reproduction in any medium, provided the original author(s) and source are credited.

\section{References}

[1] J.M. Maldacena, The large- $N$ limit of superconformal field theories and supergravity, Int. J. Theor. Phys. 38 (1999) 1113 [hep-th/9711200] [INSPIRE].

[2] S.S. Gubser, I.R. Klebanov and A.M. Polyakov, Gauge theory correlators from noncritical string theory, Phys. Lett. B 428 (1998) 105 [hep-th/9802109] [INSPIRE].

[3] E. Witten, Anti-de Sitter space and holography, Adv. Theor. Math. Phys. 2 (1998) 253 [hep-th/9802150] [INSPIRE].

[4] S.A. Hartnoll, C.P. Herzog and G.T. Horowitz, Building a Holographic Superconductor, Phys. Rev. Lett. 101 (2008) 031601 [arXiv:0803.3295] [INSPIRE].

[5] S.S. Gubser, Breaking an Abelian gauge symmetry near a black hole horizon, Phys. Rev. D 78 (2008) 065034 [arXiv:0801.2977] [INSPIRE].

[6] S.A. Hartnoll, C.P. Herzog and G.T. Horowitz, Holographic Superconductors, JHEP 12 (2008) 015 [arXiv:0810.1563] [INSPIRE].

[7] D. Musso, Introductory notes on holographic superconductors, PoS (Modave 2013) 004 [arXiv: 1401.1504] [INSPIRE].

[8] R.-G. Cai, L. Li, L.-F. Li and R.-Q. Yang, Introduction to Holographic Superconductor Models, Sci. China Phys. Mech. Astron. 58 (2015) 060401 [arXiv: 1502.00437] [INSPIRE].

[9] X.-H. Ge, B. Wang, S.-F. Wu and G.-H. Yang, Analytical study on holographic superconductors in external magnetic field, JHEP 08 (2010) 108 [arXiv:1002.4901] [INSPIRE].

[10] R. Banerjee, S. Gangopadhyay, D. Roychowdhury and A. Lala, Holographic s-wave condensate with nonlinear electrodynamics: A nontrivial boundary value problem, Phys. Rev. D 87 (2013) 104001 [arXiv:1208.5902] [InSPIRE].

[11] D. Momeni, M. Raza and R. Myrzakulov, More on Superconductors via Gauge/Gravity Duality with Nonlinear Maxwell Field, J. Grav. 2013 (2013) 782512 [arXiv:1305.3541] [INSPIRE].

[12] C.-M. Chen and M.-F. Wu, An Analytic Analysis of Phase Transitions in Holographic Superconductors, Prog. Theor. Phys. 126 (2011) 387 [arXiv:1103.5130] [INSPIRE].

[13] H.-B. Zeng, X. Gao, Y. Jiang and H.-S. Zong, Analytical Computation of Critical Exponents in Several Holographic Superconductors, JHEP 05 (2011) 002 [arXiv: 1012.5564] [INSPIRE].

[14] R.-G. Cai, H.-F. Li and H.-Q. Zhang, Analytical Studies on Holographic Insulator/Superconductor Phase Transitions, Phys. Rev. D 83 (2011) 126007 [arXiv:1103.5568] [INSPIRE].

[15] Q. Pan, B. Wang, E. Papantonopoulos, J. Oliveira and A.B. Pavan, Holographic Superconductors with various condensates in Einstein-Gauss-Bonnet gravity, Phys. Rev. D 81 (2010) 106007 [arXiv:0912.2475] [inSPIRE].

[16] Q. Pan and B. Wang, General holographic superconductor models with Gauss-Bonnet corrections, Phys. Lett. B 693 (2010) 159 [arXiv: 1005.4743] [INSPIRE]. 
[17] H.-F. Li, R.-G. Cai and H.-Q. Zhang, Analytical Studies on Holographic Superconductors in Gauss-Bonnet Gravity, JHEP 04 (2011) 028 [arXiv:1103.2833] [INSPIRE].

[18] R.-G. Cai, Z.-Y. Nie and H.-Q. Zhang, Holographic p-wave superconductors from Gauss-Bonnet gravity, Phys. Rev. D 82 (2010) 066007 [arXiv:1007.3321] [InSPIRE].

[19] L. Barclay, R. Gregory, S. Kanno and P. Sutcliffe, Gauss-Bonnet Holographic Superconductors, JHEP 12 (2010) 029 [arXiv: 1009.1991] [INSPIRE].

[20] R.-G. Cai, L. Li and L.-F. Li, A Holographic P-wave Superconductor Model, JHEP 01 (2014) 032 [arXiv: 1309.4877] [INSPIRE].

[21] Q. Pan, J. Jing and B. Wang, Analytical investigation of the phase transition between holographic insulator and superconductor in Gauss-Bonnet gravity, JHEP 11 (2011) 088 [arXiv: 1105.6153] [INSPIRE].

[22] S. Kanno, A Note on Gauss-Bonnet Holographic Superconductors, Class. Quant. Grav. 28 (2011) 127001 [arXiv:1103.5022] [INSPIRE].

[23] A. Dey, S. Mahapatra and T. Sarkar, Generalized Holographic Superconductors with Higher Derivative Couplings, JHEP 06 (2014) 147 [arXiv:1404.2190] [INSPIRE].

[24] R. Gregory, S. Kanno and J. Soda, Holographic Superconductors with Higher Curvature Corrections, JHEP 10 (2009) 010 [arXiv:0907.3203] [INSPIRE].

[25] Z. Zhao, Q. Pan, S. Chen and J. Jing, Notes on holographic superconductor models with the nonlinear electrodynamics, Nucl. Phys. B 871 (2013) 98 [arXiv:1212.6693] [INSPIRE].

[26] J. Jing, Q. Pan and S. Chen, Holographic Superconductor/Insulator Transition with logarithmic electromagnetic field in Gauss-Bonnet gravity, Phys. Lett. B 716 (2012) 385 [arXiv: 1209.0893] [INSPIRE].

[27] D. Roychowdhury, Effect of external magnetic field on holographic superconductors in presence of nonlinear corrections, Phys. Rev. D 86 (2012) 106009 [arXiv:1211.0904] [INSPIRE].

[28] S. Gangopadhyay, Holographic superconductors in Born-Infeld electrodynamics and external magnetic field, Mod. Phys. Lett. A 29 (2014) 1450088 [arXiv:1311.4416] [INSPIRE].

[29] C. Lai, Q. Pan, J. Jing and Y. Wang, On analytical study of holographic superconductors with Born-Infeld electrodynamics, Phys. Lett. B 749 (2015) 437 [arXiv:1508.05926] [INSPIRE].

[30] D. Ghorai and S. Gangopadhyay, Higher dimensional holographic superconductors in Born-Infeld electrodynamics with back-reaction, Eur. Phys. J. C 76 (2016) 146 [arXiv: 1511.02444] [INSPIRE].

[31] P. Chaturvedi and G. Sengupta, p-wave Holographic Superconductors from Born-Infeld Black Holes, JHEP 04 (2015) 001 [arXiv: 1501.06998] [inSPIRE].

[32] S. Gangopadhyay and D. Roychowdhury, Analytic study of properties of holographic superconductors in Born-Infeld electrodynamics, JHEP 05 (2012) 002 [arXiv:1201.6520] [INSPIRE].

[33] W. Yao and J. Jing, Analytical study on holographic superconductors for Born-Infeld electrodynamics in Gauss-Bonnet gravity with backreactions, JHEP 05 (2013) 101 [arXiv: 1306.0064] [INSPIRE].

[34] J. Jing, L. Wang, Q. Pan and S. Chen, Holographic Superconductors in Gauss-Bonnet gravity with Born-Infeld electrodynamics, Phys. Rev. D 83 (2011) 066010 [arXiv:1012.0644] [INSPIRE]. 
[35] S. Dey and A. Lala, Holographic s-wave condensation and Meissner-like effect in Gauss-Bonnet gravity with various non-linear corrections, Annals Phys. 354 (2014) 165 [arXiv: 1306.5137] [INSPIRE].

[36] R. Gregory, S. Kanno and J. Soda, Holographic Superconductors with Higher Curvature Corrections, JHEP 10 (2009) 010 [arXiv:0907.3203] [INSPIRE].

[37] J. Jing, Q. Pan and S. Chen, Holographic Superconductors with Power-Maxwell field, JHEP 11 (2011) 045 [arXiv: 1106.5181] [INSPIRE].

[38] J. Jing, L. Jiang and Q. Pan, Holographic superconductors for the Power-Maxwell field with backreactions, Class. Quant. Grav. 33 (2016) 025001 [InSPIRE].

[39] R.-G. Cai, Gauss-Bonnet black holes in AdS spaces, Phys. Rev. D 65 (2002) 084014 [hep-th/0109133] [INSPIRE].

[40] S. Gangopadhyay and D. Roychowdhury, Analytic study of properties of holographic superconductors in Born-Infeld electrodynamics, JHEP 05 (2012) 002 [arXiv:1201.6520] [INSPIRE].

[41] P. Appell, Sur une formule de M. Tisserand et sur les fonctions hypergéométriques de deux variables, J. Math. Pures Appl. 10 (1884) 407.

[42] S.S. Gubser and S.S. Pufu, The Gravity dual of a p-wave superconductor, JHEP 11 (2008) 033 [arXiv:0805.2960] [INSPIRE].

[43] S.A. Hartnoll, Lectures on holographic methods for condensed matter physics, Class. Quant. Grav. 26 (2009) 224002 [arXiv:0903.3246] [INSPIRE].

[44] S. Gangopadhyay and D. Roychowdhury, Analytic study of Gauss-Bonnet holographic superconductors in Born-Infeld electrodynamics, JHEP 05 (2012) 156 [arXiv:1204.0673] [INSPIRE].

[45] S. Gangopadhyay and D. Roychowdhury, Analytic study of properties of holographic superconductors in Born-Infeld electrodynamics, JHEP 05 (2012) 002 [arXiv:1201.6520] [INSPIRE].

[46] R.-G. Cai, Z.-Y. Nie and H.-Q. Zhang, Holographic p-wave superconductors from Gauss-Bonnet gravity, Phys. Rev. D 82 (2010) 066007 [arXiv: 1007.3321] [INSPIRE]. 